\title{
Cold atom simulation of interacting relativistic quantum field theories
}

\author{
J. Ignacio Cirac ${ }^{1}$, Paolo Maraner ${ }^{2}$ and Jiannis K. Pachos ${ }^{3 *}$ \\ ${ }^{1}$ Max-Planck-Institut für Quantenoptik, Hans-Kopfermann-Str. 1, D-85748 Garching, Germany \\ ${ }^{2}$ School of Economics and Management, Free University of Bozen-Bolzano, via Sernesi 1, 39100 Bolzano, Italy and \\ ${ }^{3}$ School of Physics and Astronomy, University of Leeds, Woodhouse Lane, Leeds LS2 9JT, UK
}

(Dated: October 24, 2018)

\begin{abstract}
We demonstrate that Dirac fermions self-interacting or coupled to dynamic scalar fields can emerge in the low energy sector of designed bosonic and fermionic cold atom systems. We illustrate this with two examples defined in two spacetime dimensions. The first one is the self-interacting Thirring model. The second one is a model of Dirac fermions coupled to a dynamic scalar field that gives rise to the Gross-Neveu model. The proposed cold atom experiments can be used to probe spectral or correlation properties of interacting quantum field theories thereby presenting an alternative to lattice gauge theory simulations.
\end{abstract}

PACS numbers: 11.15.-q, 11.15.Ha

Introduction:- To date there exist several important open problems in quantum field theories (QFTs) ranging from the convergence of the scattering matrix to the infrared behaviour of quantum chromodynamics (QCD). Such problems can be probed analytically only by nonperturbative methods that seem to be intractable in three and four dimensions. As an alternative, numerical techniques or quantum simulations can play a central role in obtaining insight into the Standard Model.

Recently, much interest is focused on simulating QFTs with cold atoms [1 [5]. In the relativistic domain, these studies are usually limited to generating Dirac fermions [6, 7] and background fields 8 $8-10$. In this letter, we propose the realisation of Dirac fermions interacting with dynamic fields by employing cold atoms in optical lattices. This constitutes the first step towards the quantum simulation of a general model with coupled relativistic fermionic and bosonic fields. Cold atoms provide a controlled medium with well understood interactions. A wide range of quantum optics and atomic physics technology allows for the preparation, manipulation and detection of a variety of interesting many body phenomena. Employing cold atoms gives us the possibility to consider two, three and four spacetime dimensions, to tune the couplings of the interactions and to explore the behavior of multi-component fields. Compared to numerical simulations of lattice gauge theories on computers, a physical simulation on a quantum system naturally overcomes the sign problem [11.

Here we show how to simulate a two-dimensional self-interacting model of Dirac fermions, known as the Thirring model [12, 13] and two-dimensional Dirac fermions coupled to a scalar field that is equivalent to the Gross-Neveu model. The Hamiltonians of these systems are supported on one spatial dimension. The neces-

*Email: j.k.pachos@leeds.ac.uk sary building blocks are the Dirac Hamiltonian, which describes relativistic fermions, and the interaction of fermions with themselves or with a dynamic scalar field. This goes beyond previous proposals concerned with dynamical fermions coupled to classical fields. We show how the required components naturally emerge in the low energy sector of specifically designed lattice Hamiltonians. The Dirac operator describes the continuum limit of certain fermionic lattices [8, 14, 15, as in graphene [16], that remarkably give rise to both the spin and the linear dispersion relation. Compared to previous approaches [8] here we realise the Dirac operator only by the free tunnelling of single species of atoms. Slow spatial variations of the lattice couplings result, in the Dirac picture, to an interaction with a scalar field background [10. When these distortions are caused by coupling the lattice fermions to bosons, the resulting fields become dynamic. Self-energy terms can be implemented giving rise to a variety of interesting QFTs. The presented models are exactly solvable and serve for demonstrating the ability to simulate important properties of the Standard Model such as dynamical symmetry breaking and mass generation with cold atoms.

Thirring model:- Our starting point is the twodimensional Thirring model [12, 13]. It describes interacting fermions with the Hamiltonian

$$
\frac{H_{\mathrm{T}}}{\hbar}=\int d x\left(v_{\mathrm{s}} \bar{\Psi} \gamma_{1} p \Psi+\frac{m_{0} v_{\mathrm{s}}^{2}}{\hbar} \bar{\Psi} \Psi+\frac{g}{2} \bar{\Psi} \gamma^{\mu} \Psi \bar{\Psi} \gamma_{\mu} \Psi\right) .
$$

Here the $\gamma^{\prime}$ 's are two-dimensional matrices satisfying $\left\{\gamma_{\mu}, \gamma_{\nu}\right\}=2 \eta_{\mu \nu}$ for $\mu, \nu=0,1$ with $\eta_{\mu \nu}=\operatorname{diag}(1,-1)$, $\gamma_{5}=\gamma_{0} \gamma_{1}$ and $\Psi$ is a two-dimensional spinor with $\bar{\Psi}=\Psi^{\dagger} \gamma_{0}$. The mass of the fermions is $m_{0}, g$ is their dimensionless self-interaction coupling constant and $v_{\mathrm{s}}$ is the sound velocity taken in high energy to be the speed of light. This model has exciting physics with the massless case, $m_{0}=0$, being equivalent to free bosons and the massive case being equivalent to the Sine-Gordon model [17. 


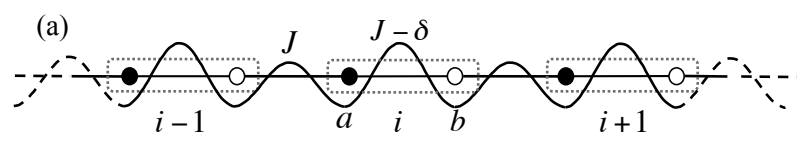

(b)

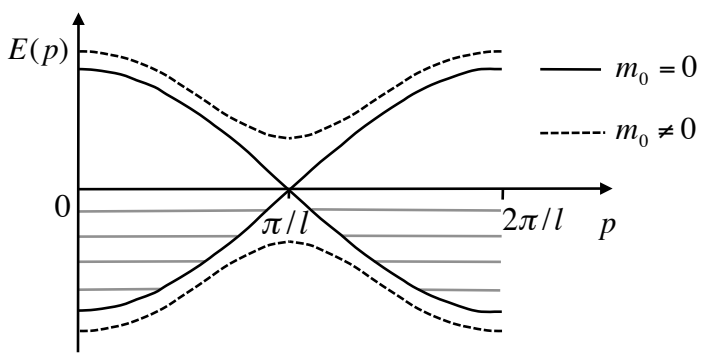

FIG. 1: (a) The one-dimensional superlattice with tunnelling fermionic atoms that simulates Dirac fermions. Each unit cell includes two fermion sites, $a$ and $b$. An alternating distortion of the tunnelling couplings $J$ and $J-\delta$ gives rise to the mass term, $\delta$. (b) The energy dispersion relation as a function of momentum, $p$. At half filling and for $\delta=0$ the low energy behaviour is linear with respect to $p$, allowing the Dirac operator description.

To simulate the Thirring model in an atomic system we consider a linear bicolourable fermionic lattice with spacing $l$ (see Fig. 1(a)) which is subject to the Hubbard Hamiltonian

$$
\begin{aligned}
\frac{H}{\hbar}=2 \pi \sum_{i}[ & -J\left(a_{i}^{\dagger} b_{i}+b_{i}^{\dagger} a_{i+1}\right)+\delta a_{i}^{\dagger} b_{i}+\text { H.c. } \\
& \left.+U a_{i}^{\dagger} a_{i} b_{i}^{\dagger} b_{i}\right] .
\end{aligned}
$$

Here $J$ is the tunnelling coupling between neighbouring sites of the lattice of the same fermionic atoms $a$ and $b$ with $\left\{a_{i}, a_{j}^{\dagger}\right\}=\left\{b_{i}, b_{j}^{\dagger}\right\}=\delta_{i j}$ and all the other anticommutators vanishing. The $a, b$ index is a spatial distinction within the unit cell that allows the encoding of the spin degree of freedom. The tunnelling distortion $\delta$ occurs on alternating links, as shown in Fig. 1(a), while $U$ is the interactions coupling between fermions in the same cell.

If we diagonalise the $J$-term of the Hamiltonian in the $a, b$ basis we find the dispersion relation $E_{ \pm}(p)=$ $\pm 2\left|\cos \frac{p l}{2}\right|$ plotted in Fig. 1 (b). It can be easily seen that there is a single Fermi point, $P=\pi / l$, for which $E_{ \pm}(P)=$ 0 . If the lattice is half filled with fermions, which occupies the valence band completely, the behaviour of the small energy fluctuations is governed by the Hamiltonian expanded around $P$. Setting $p=P+k$ for $|k| \ll 1 / l$ we obtain a dispersion that is linear in momentum, $k$. Hence, the fermionic tunnelling term around the Fermi point can be efficiently described by the relativistic Dirac Hamiltonian $J l \int d k \psi_{k}^{\dagger} \sigma_{2} k \psi_{k}$, where $\psi=(a, b)^{T}$ corresponds to the one-dimensional version of the Kogut-Susskind fermions [14, 15]. To assign the appropriate dimensions to the Dirac fermions we set $\psi=\sqrt{2 l} \Psi$. In this way we obtain the massless free Dirac fermion term of (1) written in the momentum representation with $\gamma_{0}=\sigma_{1}, \gamma_{1}=i \sigma_{3}$

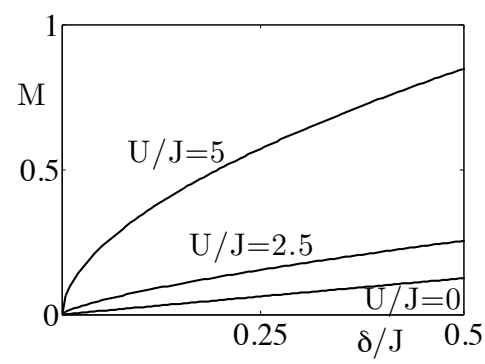

FIG. 2: The regularised mass $M$, in units of $\hbar /\left(v_{\mathrm{s}} l\right)$, as a function of the tunnelling disorder $\delta$ for various interaction strengths $U$. When no interactions are present the mass increases, as expected, linearly as a function of $\delta$. The presence of interactions dramatically changes this behaviour even for moderate ratios of $U / J$.

and $v_{\mathrm{s}}=2 \pi \cdot 2 l J$. It can be verified that the continuum limit of (2) gives also rise to the rest of the terms in (1) with $m_{0} v_{\mathrm{s}}^{2} / \hbar=2 \pi \cdot 2 \delta$ and $g=2 \pi \cdot 2 U l$. The continuum limit corresponds to small lattice spacing. This is equivalent to restricting to the low energy sector of the system where states have a large wavelength support. These are exactly the states we are interested in for probing the infrared behaviour of QFT, such as the ground state and its gapped or gapless nature.

The Hamiltonian (2) can be realised with cold atoms as follows. The one-dimensional fermionic tunnelling term of 22 appears when a fermionic gas is placed in an optical lattice with very tight confinement in the other two directions. The $\delta$-term corresponds to a uniformly decreased tunnelling coupling between sites of the same cell. It can be generated, e.g. by employing superlattices as seen in Fig. 1(a). This alternatively signifies that inhomogeneity in the tunnelling coupling due to experimental imperfections will generate a mass term as observed in [1]. The final $U$-term results from the interaction between the atoms $a$ and $b$ present in the same cell [See supplementary material at [URL will be inserted by AIP]].

The Thirring model is the simplest relativistic interacting QFT that one could implement in the laboratory with present technology. Its realisation can demonstrate the renormalisation of mass due to interactions. Indeed, $m_{0} \neq 0$ is the fermionic mass of the classical theory. When the interactions $g$ are introduced then a regularisation condition needs to be adopted, $|p|<\Lambda$, where $\Lambda$ is a momentum cutoff that excludes modes with unphysically high energy. Our system is naturally regularised due to the underlining lattice structure, where the momentum cutoff is related to the optical lattice spacing by, $\Lambda=\pi / l$. A quantum field theory is called renormalisable when this cutoff can be absorbed in the initial, bare parameters of the model, such as the mass. The massive Thirring model is such a renormalisable theory where the 
interactions $g$ give rise to the following regularised mass

$$
\frac{M v_{\mathrm{s}}}{\hbar}=\frac{\delta}{l} \frac{\tan \frac{\pi U}{\pi J+U}}{U} \exp \left(\frac{U}{\pi J+U} \sinh ^{-1} \frac{\pi J}{\delta}\right) .
$$

The corresponding coupling regime $0 \leq U / J<\pi$ is well within the realm of current experiments [18]. This effective mass is exactly the energy gap above the ground state obtained for zero momentum excitations, while nonzero momenta give a continuum spectrum above the gap. Fixing the lattice spacing, $l$, according to the wavelength of the optical lattice, one can plot the effective mass as a function of the atomic parameters $\delta$ and $U$, as seen in Figure 2, Current experiments routinely probe such excitation gaps in cold atom systems 1 .

The atomic simulation relies on direct exchange of atoms, while the value of $M$ increases when the couplings $U$ or $\delta$ increase, in a non-perturbative way, or when the lattice spacing $l$ decreases. The size of the gap can, hence, be efficiently controlled with a variety of experimental means which alleviates the low temperature requirements [19]. This facilitates the experimental measurement of the gap by a spectral analysis of the atomic system [1]. The renormalisation step would then necessitate to take the 'bare' mass $m_{0}$ decrease as a function of $\Lambda$ so that a finite constant value of the 'physical' mass is obtained. It is intriguing that this renormalisation procedure can be established experimentally by studying the spectral behaviour of the fermionic lattice system. Observing such a strong renormalisation of parameters (see Fig. 2 in a system of cold atomic gases provides a unique fingerprint of strong correlations.

Fermion-scalar interaction and the GrossNeveu model:- Next we consider a two-dimensional model where an $N$-colour massless Dirac fermion $\Psi_{n}$ $n=1, \ldots, N$ interacts with a massive quantised scalar field $\Phi$ according to the Hamiltonian

$$
\frac{H_{\Phi}}{\hbar}=\int d x\left(v_{\mathrm{s}} \bar{\Psi}_{n} \gamma_{1} p \Psi_{n}+g m \Phi \bar{\Psi}_{n} \Psi_{n}+\frac{m^{2}}{2} \Phi^{2}\right) .
$$

Here we assume summation over the colour index $n, g$ is the coupling strength between the bosonic and fermionic fields and $m$ is a mass scale that can be absorbed in $\Phi$. It can be shown that this Hamiltonian yields exactly equivalent fermionic behavior as the Gross-Neveu model 20. given by

$$
\frac{H_{\mathrm{GN}}}{\hbar}=\int d x\left[v_{\mathrm{s}} \bar{\Psi}_{n} \gamma_{1} p \Psi_{n}+\frac{g^{2}}{2}\left(\bar{\Psi}_{n} \Psi_{n}\right)^{2}\right] .
$$

The Hamiltonian $H_{\mathrm{GN}}$ describes massless fermions subject to attractive interactions. This attraction causes the fermions to create bosonic pairs. Eventually, these composite bosons condense breaking spontaneously the $Z_{2}$ symmetry, $\Psi \rightarrow \gamma_{5} \Psi$, of Hamiltonian (5), thereby causing the fermions to dynamically acquire mass. This fascinating property is very similar to the behaviour of the
BCS theory of superconductivity or of four-dimensional QCD. Note that (4) does not contain a kinetic term for the scalar field. This corresponds to a Yukawa theory with interactions mediated by infinitely massive fields, making their propagation point-like and resulting in the Gross-Neveu effective four-fermion interaction.

We will now consider a cold atom system that gives rise to $H_{\Phi}$ [21, which would make it possible to observe the dynamical mass generation experimentally. The kinetic term of the Dirac fermions, $\Psi_{n}$, can be produced by the same fermionic tunnelling term as in (2). In general, a variety of interaction terms can be generated between bosonic and fermionic atoms. To conform with (4), we specifically want the bosonic modes, $\Phi$, to couple linearly to the fermionic ones, $\Psi_{n}$, as dictated by the minimal coupling prescription. Such an interaction can result similarly to the $m_{0}$-term of the Thirring model. A position dependent tunnelling distortion gives rise to a classical scalar field configuration. Formally, the quantisation of the scalar field is obtained by writing it as $\Phi=\left(d^{\dagger}+d\right) / \sqrt{2}$, where $d$ is a bosonic mode. Substituting it into $H_{\Phi}$ gives the quantised Dirac fermion-scalar model. In the cold atom setting, this can be achieved by employing a bosonic condensate interacting with the lattice fermions as we shall see below.

Consider a one-dimensional fermionic lattice superposed with a one-dimensional bosonic lattice, as seen in Fig. 3. We assume that the dynamics of the atoms is described by

$$
\begin{gathered}
\frac{H}{\hbar}=2 \pi \sum_{i}\left\{\left[-\left(J^{\prime} a_{n, i}^{\dagger} b_{n, i}+J b_{n, i}^{\dagger} a_{n, i+1}\right)-\right.\right. \\
\left.\left.\delta \alpha_{i}^{\dagger} \alpha_{i} a_{n, i}^{\dagger} b_{n, i}+\text { H.c. }\right]+U \alpha_{i}^{\dagger^{2}} \alpha_{i}^{2}+\mu \alpha_{i}^{\dagger} \alpha_{i}\right\} .
\end{gathered}
$$

Here the $a_{n}$ 's and $b_{n}$ 's are $N$ different species of fermionic atoms (summation over $n$ is assumed) and $\alpha_{i}=D+$ $d_{i}$ is an atomic condensate with particle density $D$ and bosonic operator $d$. When the couplings of (6) and the condensate density are appropriately tuned, then the low energy behaviour of the Hamiltonian $H$ reproduces $H_{\Phi}$. Indeed, for $J^{\prime}=J-\delta D^{2}$ and $\mu=-2 D^{2} U$ one reproduces the desired low energy behavior with $g^{2}=2 \pi \cdot 2 \delta^{2} l / U$ and $v_{\mathrm{s}}=2 \pi \cdot 2 J l$. To suppress spurious terms we take the weak fluctuation limit $\left\langle d^{\dagger} d\right\rangle \ll D^{2}$.

The terms in (6) can be realised in the lattice configuration of Fig. 3 by employing one-dimensional optical lattices filled with the appropriate species of atoms. All the required interactions naturally appear in cold atom settings and can be tuned, e.g. by Feshbach resonances. The $J$ - and $J^{\prime}$-terms of (6) result from the tunnelling of the fermionic atoms along their lattice and contribute to the free Dirac fermion propagation. The $\delta$-term results from the interaction between bosonic and fermionic atoms that generate a fermionic tunnelling controlled by the bosonic populations. It gives rise to the interaction 


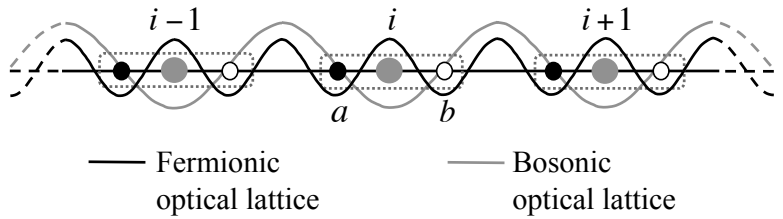

FIG. 3: The one-dimensional optical lattice with tunnelling fermions and bosons that simulates the Dirac fermion-scalar field model. The bosonic sites are placed in-between the fermionic ones with double spacing. In this way the bosonic population on site $i$ controls the fermionic tunnelling within the same cell.

between the scalar and the fermion fields. In (6) there is no tunnelling term for the bosonic atoms. Hence, the required system comprises of independent BECs at each site of a bosonic lattice [22, 23]. The $U$-term describes the interaction between bosonic atoms on the same site and the $\mu$-term is given by their chemical potential. Importantly, small errors in the values of all of these couplings result in modifications of the parameters of the effective Hamiltonian (4) or the generation of similar terms [10]. The persistence of the effective Dirac description in the low energy limit is a characteristic also present in the Thirring model simulation.

It has been shown [20] that the initially massless fermions of the Gross-Neveu model dynamically acquire an effective mass. In terms of the atomic parameters, the emerging mass gap is given by

$$
\frac{M v_{\mathrm{s}}}{\hbar}=\frac{\pi}{l} \exp \left(-\frac{\pi}{N} \frac{J U}{\delta^{2}}\right)
$$

This result is exact in the large $N$ limit but is expected to be a good approximation even for moderate, experimentally feasible, values of $N$ of the order of 2 or 3 . While the mass, $M$, goes to infinity when $l$ goes to zero, the renormalisation prescription absorbs the infinity in the 'bare' coupling $g$. In the case of the optical lattice realisation we do not need to renormalise the coupling $g$ as we are working with a fixed lattice spacing $l$. Such a simulation can detect the dynamical generation of mass and verify the predicted behaviour (7) as a function of the atomic couplings.

Conclusions:- The above method can be straightforwardly generalised to other more complex QFTs. For example, to simulate quantum electrodynamics in two spacetime dimensions $\left(\mathrm{QED}_{2}\right)$ we need a similar atomic setting to the fermion-scalar model described above. Apart from the addition of a kinetic term for the bosonic field we need to impose the Gauss constraint in the atomic level. An atomic model that can give rise to $\mathrm{QED}_{2}$ as well as its generalisation to four dimensions will be presented elsewhere.

Going beyond QED one could realise the $\mathrm{SU}(N)$ YangMills gauge field coupled to an $N$-colour Dirac fermion (see (4)) that gives rise to QCD. The challenge faced at this point is to realise the higher order bosonic selfinteraction terms that are necessary to simulate the nonlinear behaviour of the Yang-Mills theory. It is a fascinating perspective to probe the generation of a mass gap in such a QCD simulation. In parallel, a wealth of possibilities opens for the simulation of supersymmetric QFTs or combining QFT and relativity in an atomic system. It is highly plausible that the experimental realisation of the simple models presented here provide a unique probing tool into the open questions of interacting relativistic QFTs.

Acknowledgements:- J.K.P. would like to thank N. Cooper, J. Dunningham and S. Ruijsenaars for inspiring discussions. This work was supported by the Royal Society. J.I.C. acknowledges funding from the EU (project AQUTE) and the DFG (Forchergruppe 635).

[1] E. Haller, R. Hart, M. J. Mark, J. G. Danzl, L. Reichsöllner, M. Gustavsson, M. Dalmonte, G. Pupillo and H.-C. Nägerl, arXiv:1004.3168 (2010).

[2] H. P. Büchler, G. Blatter, and W. Zwerger, Phys. Rev. Lett. 90, 130401 (2003).

[3] M. Kormos, G. Mussardo, and A. Trombettoni, Phys. Rev. A 81, 043606 (2010); L. Lepori, G. Mussardo, A. Trombettoni, arXiv:1004.4744 (2010).

[4] F. L. Semiao, and M. Paternostro, arXiv:1005.2775 (2010).

[5] A. Bermudez, et al., arXiv:1004.5101 (2010).

[6] R. Gerritsma, G. Kirchmair, F. Zähringer, E. Solano, R. Blatt and C. F. Roos, Nature 463, 68 (2010).

[7] J. Y. Vaishnav and C. W. Clark, Phys. Rev. Lett. 100, 153002 (2008).

[8] J. Ruostekoski, G. V. Dunne, and J. Javanainen, Phys. Rev. Lett. 88, 180401 (2002); J. Ruostekoski, J. Javanainen, and G. V. Dunne, Phys. Rev. A 77, 013603 (2008).

[9] N. Goldman, A. Kubasiak, A. Bermudez, P. Gaspard, M. Lewenstein and M. A. Martin-Delgado, Phys. Rev. Lett. 103, 035301 (2009).

[10] P. Maraner and J. K. Pachos, Phys. Lett. A 373, 2616 (2009).

[11] S. A. Baeurle, Phys. Rev. Lett. 89, 080602 (2002).

[12] W. Thirring, Annals Phys. 3, 91 (1958).

[13] V. E. Korepin, N. M. Bogoliubov, and A. G. Izergin, Quantum inverse scattering method and correlation functions, Cambridge Univ. Press, Cambridge (1993).

[14] J. B. Kogut, and L. Susskind, Phys. Rev. D 11, 395 (1975); L. Susskind, Phys. Rev. D 16, 3031 (1977).

[15] R. Jackiw and C. Rebbi, Phys. Rev. D 13, 3398 (1976).

[16] A. H. Castro Neto, F. Guinea, N. M. R. Peres, K. S. Novoselov and A. K. Geim, Rev. Mod. Phys. 81, 109 (2009); R. Jackiw, and S.-Y. Pi, Phys. Rev. Lett. 98, 266402 (2007).

[17] S. Coleman, Phys. Rev. D 11, 2088 (1975).

[18] R. Jördens, N. Strohmaier, K. Günter, H. Moritz and T. Esslinger, Nature 455, 204 (2008).

[19] S. Trotzky et al., Science 319, 295 (2008).

[20] D. J. Gross and A. Neveu, Phys. Rev. D 10, 3225 (1974). 
[21] Hamiltonian 44 is significantly simpler to simulate than the actual Gross-Neveu one (5) as the interaction term in the latter has a summation over the fermionic components squared that demands the fine tunning of many atomic interactions.
[22] V. Schweikhard, S. Tung, and E. A. Cornell, Phys. Rev. Lett. 99, 030401 (2007).

[23] Z. Hadzibabic, S. Stock, B. Battelier, V. Bretin, and J. Dalibard, Phys. Rev. Lett. 93, 180403 (2004). 